\title{
AUTHENTICATION OF PATIN (PANGASIUS MICRONEMUS) FISH OIL ADULTERATED WITH PALM OIL USING FTIR SPECTROSCOPY COMBINED WITH CHEMOMETRICS
}

\author{
ANGGITA ROSIANA PUTRI ${ }^{1}$, ABDUL ROHMAN ${ }^{1,2^{*}}$, SUGENG RIYANTO ${ }^{1}$ \\ 1Faculty of Pharmacy, Gadjah Mada University, Yogyakarta, 55281, Indonesia, ${ }^{2}$ Research Center of Halal Products, Gadjah Mada \\ University, Yogyakarta, 55281 Indonesia \\ Email: abdul_kimfar@ugm.ac.id
}

Received: 23 Nov 2018, Revised and Accepted: 06 Apr 2019

\begin{abstract}
Objective: The goal of this research was to perform authentication of patin (Pangasius micronemus) fish oil (PFO) adulterated with palm oil (P0) using FTIR spectroscopy combined with chemometrics method.

Methods: Patin fish oil (PFO) and PFO adulterated with palm oil (PO) were measured using FTIR instrument at wavenumbers region of $4000-650 \mathrm{~cm}^{-1}$. The chemometrics methods, namely multivariate calibration of partial least square (PLS) and principal component regression (PCR) were used to make calibration and validation models during quantification. Discriminant analysis (DA) was used to make grouping pure PFO and PFO adulterated with PO.

Results: The results showed that PLS and PCR could be used to quantify PO as adulterant in PFO, either in calibration or validation models. FTIR spectroscopy combined with multivariate calibration offered accurate and precise method for quantitative analysis with $\mathrm{R}^{2}$ value of $>0.999$ and low
\end{abstract} RMSEC and RMSEP. DA was capable of grouping PFO and PFO adulterated with PO with an accuracy level of $100 \%$.

Conclusion: FTIR spectroscopy combined with chemometrics could be reliable technique for quantification and discrimination of PFO and PFO adulterated with PO.

Keywords: Patin fish oil, Palm Oil, Authentication, Partial least square, Principle component regression, Discriminant analysis

(C) 2019 The Authors. Published by Innovare Academic Sciences Pvt Ltd. This is an open-access article under the CC BY license (http://creativecommons.org/licenses/by/4.0/) DOI: http://dx.doi.org/10.22159/ijap.2019v11i3.30947

\section{INTRODUCTION}

Patin fish (Pangasius micronemus) is the one of the most popular freshwater fish in Indonesia. Patin fish oil (PFO) has the potency to be used as a source of omega- 3 fatty acids, which are beneficial to human health. Omega- 3 fatty acids are unsaturated fatty acid which consist of eicosapentanoic acid (EPA) and docosahexanoic acid (DHA). Omega-3 fatty acids can be used to prevent some diseases such as hypertension, eczema, psoriasis, osteoporosis, breast cancer, allergic [1], cardiovascular [2], and Alzheimer [3].

Oils might have the same composition with other oils, therefore it is possible to adulterate high priced oil with lower ones [4]. Authentication is important to protect the consumer from adulteration practices. Patin fish oil (PFO) has the possibility to be adulterated with other oils which are cheaper like palm oil so it will decrease the quality of PFO. Some authentication methods based on physicochemical properties and molecular biology have been developed for authentication analysis, and among this method, fourier transform infrared (FTIR) spectroscopy combined with chemometrics is the most used ones. Because of its nature as a fingerprint technique, meaning that every oils have different FTIR spectra, FTIR spectroscopy is an idela method [5].

Chemometrics is the method in chemical that using mathematical, statistical, and other methods to make a measurement model by analysing chemical data [6]. FTIR Spectroscopy that combined with chemometrics are widely used to detect adulteration product like adulteration virgin olive oil with palm oil [5], adulteration in virgin coconut oil [7], sesame oil with corn oil [8], adulteration sunflower and corn oils in extra virgin olive oil [9], detection of rice bran oil as adulterant in extra virgin olive oil [10], and candlenut adulterated with palm oil [11]. FTIR spectroscopy was also used for monitoring oxidative stabilities of frying oils [12]. Chemometrics usually use multivariate calibration for analysis complex mixture. This method is rapid, no time consuming, easy, and simple preparation sample [13]. Partial least square (PLS) and principle component regression (PCR) were commonly used in multivariate calibration techniques [14]. Another method in chemometrics is discriminant analysis (DA). This method was used to find the relationship between descriptive variable and qualitative variable [15]. The goal of this research is to authenticate patin (Pangasius micronemus) fish oil (PFO) adulterated with palm oil (PO) using FTIR spectroscopy combined with chemometrics. In this research, PLS and PCR calibration techniques was used for quantitative analysis, while DA was explored for classification between pure PFO and PFO adulterated with PO.

\section{MATERIALS AND METHODS}

\section{Sample preparation}

Patin Fish was obtained from freshwater fish cultivation in Tulungagung, East Java, Indonesia. PFO was extracted with maceration process using $n$-hexane solvent. Palm oil was obtained from market in Yogyakarta, Indonesia. Twenty (21) samples containing PFO and PO with the concentration range of $0-100 \% \mathrm{v} / \mathrm{v}$ were prepared for quantitative analysis using PLS and PCR, and 13 samples containing pure PFO and PFO adulterated with PO was prepared for DA.

\section{FTIR analysis}

Samples were analysed using FTIR spectrometer (Thermo Scientific Nicolet iS10, Madison, WI). This instrument was controlled with Omnic software. The measurements were done in middle infrared region of $4000-650 \mathrm{~cm}^{-1}$ with 32 scanning and at a resolution of $16 \mathrm{~cm}^{-1}$. The background scanning has to do to reduce the effect of the reference spectrum of the air. Before and after analysis sample, ATR crystal has to be cleaned with acetone p.a. Replications were done with scanning same samples for 3 times.

\section{Chemo metrics}

Chemo metrics analysis including multivariate calibration and discriminant analysis was performed using TQ Analyst software version 9 (Thermo Fisher Scientific, Inc.). For quantification, multivariate calibrations used were PLS and PCR.

\section{RESULTS AND DISCUSSION}

\section{Spectra analysis}

Oils and fats are basically triglycerides which can be differed from the fatty acid composition in terms of length chain and unsaturation 
degree. The adulteration practice was usually met in high priced oil having similar color with oil adulterant, therefore, it would be difficult to detect the oil that mixed with another oil, physically [4]. Meanwhile, fats and oils can be differentiated using FTIR spectra due to the property of FTIR spectra as fingerprint technique. FTIR spectra could be used to get information about molecular structure from the vibration of functional groups at the certain wavenumbers [16]. The FTIR spectra of patin fish oil (PFO) and palm oil (PO) were showed in fig. 1. Each peaks and shoulders correspond to functional groups responsible for the absorption of infrared radiation. Table 1 explain the functional groups corresponding to each peaks/ shoulders. There are some differences in both spectra at wavenumber around $2954 \mathrm{~cm}^{-1}$ and at the fingerprint area $\left(1500-650 \mathrm{~cm}^{-1}\right)$. PO spectra have no peak at $2954 \mathrm{~cm}^{-1}$ and around $700 \mathrm{~cm}^{-1}$. Thus, these areas can be used for detection and quantification of PFO that adulterated with PO.

Table 1: Vibration of functional groups in patin fish oil (PFO) and palm oil at certain wavenumbers [20]

\begin{tabular}{ll}
\hline Wavenumber $\left(\mathbf{c m}^{-1}\right)$ & Functional group \\
\hline 3005 & cis-double-bond stretching \\
2954 & $-\mathrm{CH}$ 3 asymmetrical strecth \\
2922 and 2854 & Asymmetric and symmetric vibrations of methylene $\left(-\mathrm{CH}_{2}\right)$ group \\
1744 & $-\mathrm{C}=0$ stretch \\
1460 & $-\mathrm{CH}$ bending vibration of an aliphatic group \\
1374 & $-\mathrm{CH}_{3}$ bending vibration \\
1233 and 1157 & Stretching vibration of-C-O ester \\
1113 & Bending vibration of-CH \\
721 & Rocking vibration of methylene $\left(-\mathrm{CH}_{2}\right)$ \\
\hline
\end{tabular}

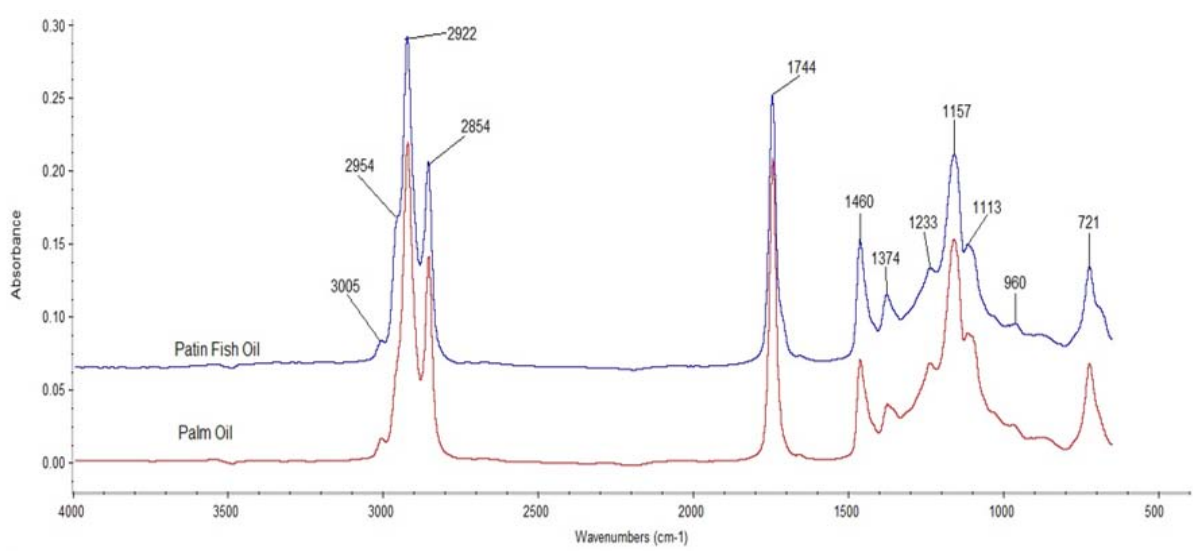

Fig. 1: FTIR spectra of patin fish oil (PFO) and palm oil (PO) at wavenumber of 4000-650 $\mathrm{cm}^{-1}$

A

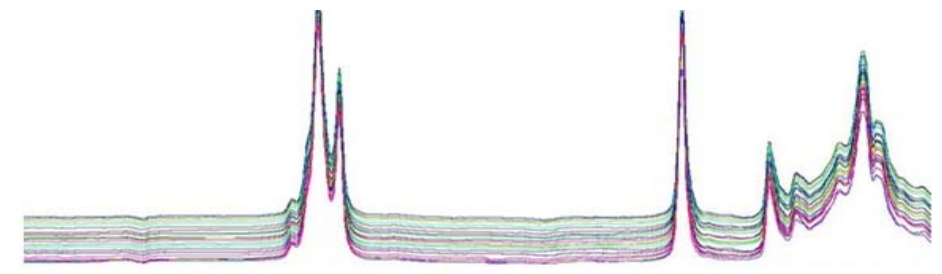

B

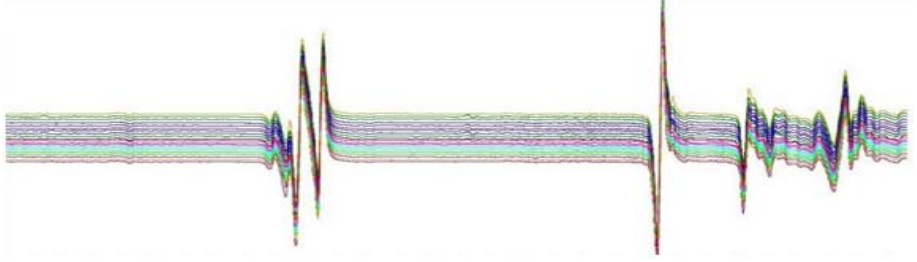

C

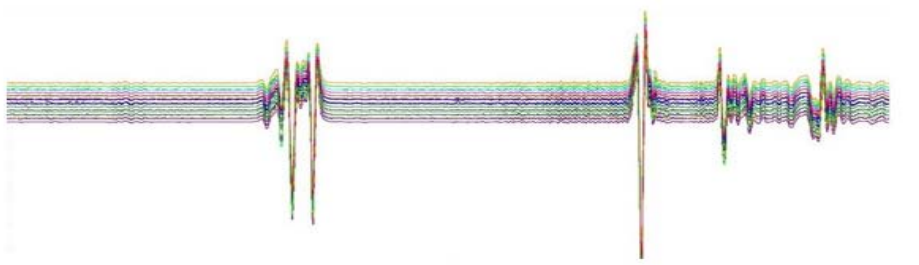

Fig. 2: Overlay FTIR spectra of patin fish oil (PFO) and palm oil (PO). (A) FTIR normal spectra (B) first derivative spectra (C) second derivative spectra 
Quantification of patin fish oil (PFO) that mixed with PO was performed with partial least square (PLS) and principle component regression (PCR). For quantification, 21 samples containing PFO that mixed with PO with range concentration $0-100 \%$ and 13 samples containing pure PFO. The quantification using spectral regions at 1001-1501, 1776-1712, and 2819-2981 $\mathrm{cm}^{-1}$. These regions was selected by optimization processes that give the highest values for $\mathrm{R}^{2}$ and the lowest values of error [7]. PLS method using correlation between spectral absorption change and concentration of samples then computing with other spectra that may disturb analyte spectra [15]. While the PCR method combines spectral and data concentration sample into a model in one step [16]. Overlay FTIR spectra of PFO and PO was shown in fig. 2. In PLS and PCR calibration techniques, linearity was used to evaluate the relationship between absorbance and samples concentration. From the PLS and PCR method, the statistical parameters namely coefficient of determination $\left(\mathrm{R}^{2}\right)$ value, root mean square error of calibration (RMSEC), and root mean square error of prediction (RMSEP) in normal, first derivative and second derivative spectra were compiled in table 2 .

Root mean square of calibration (RMSEC) is the value that used to evaluate error in calibration model and the root mean square of prediction (RMSEP) is used to evaluate the validation model [7].

Table 2: The performance of partial least square (PLS) and principle component regression (PCR) for quantitative analysis of patin fish oil (PFO) in palm oil (PO)

\begin{tabular}{|c|c|c|c|c|c|c|c|}
\hline \multirow[t]{2}{*}{ Model } & \multirow[t]{2}{*}{ Spectra } & \multicolumn{2}{|l|}{ Equation } & \multicolumn{2}{|l|}{$\mathbf{R}^{2}$} & \multirow[t]{2}{*}{ RMSEC (\%v/v) } & \multirow{2}{*}{$\begin{array}{l}\text { RMSEP } \\
(\% \mathrm{v} / \mathrm{v})\end{array}$} \\
\hline & & Calibration & Validation & Calibration & Validation & & \\
\hline \multirow[t]{3}{*}{ PLS } & Normal & $y=0.9737 x+0.2142$ & $y=0.9852 x+0.9104$ & 0.9674 & 0.9689 & 5.50 & 3.59 \\
\hline & First derivative & $y=0.9994 x+0.0268$ & $y=1.0078 x-0.7823$ & 0.9994 & 0.9985 & 0.805 & 2.22 \\
\hline & Second derivative & $y=0.9964 x+0.1433$ & $y=1.0087 x-0.8251$ & 0.9995 & 0.9983 & 0.778 & 2.43 \\
\hline \multirow[t]{3}{*}{ PCR } & Normal & $y=1.0034 x-0.0449$ & $y=1.0054 x-0.3167$ & 0.9961 & 0.9938 & 2.54 & 2.61 \\
\hline & First derivative & $y=0.9993 x+0.0334$ & $y=1.0075 x-0.7332$ & 0.9993 & 0.9985 & 0.889 & 2.07 \\
\hline & Second derivative & $y=1.0006 x-0.1186$ & $y=1.0078 x-0.7082$ & 0.9989 & 0.9980 & 1.14 & 2.18 \\
\hline
\end{tabular}

PCR and PLS calibration using first derivative FTIR spectra have the best values in terms of high $\mathrm{R}^{2}$ and low of RMSEC and RMSEP values (table 2). The first derivative spectra could identify the targeted spectra from the mixed samples by increasing the spectral resolution. The $\mathrm{R}^{2}$ values obtained using PLS and PCR calibration model were of 0.9994 and 0.9993 (fig. 3) and 0.9985 in validation model (fig. 4). The high $\mathrm{R}^{2}$ value and low values of RMSEC and RMSEP indicated that calibration and validation models using PLS and PCR were accurate and precise to measure the levels of PFO adulterated with PO.

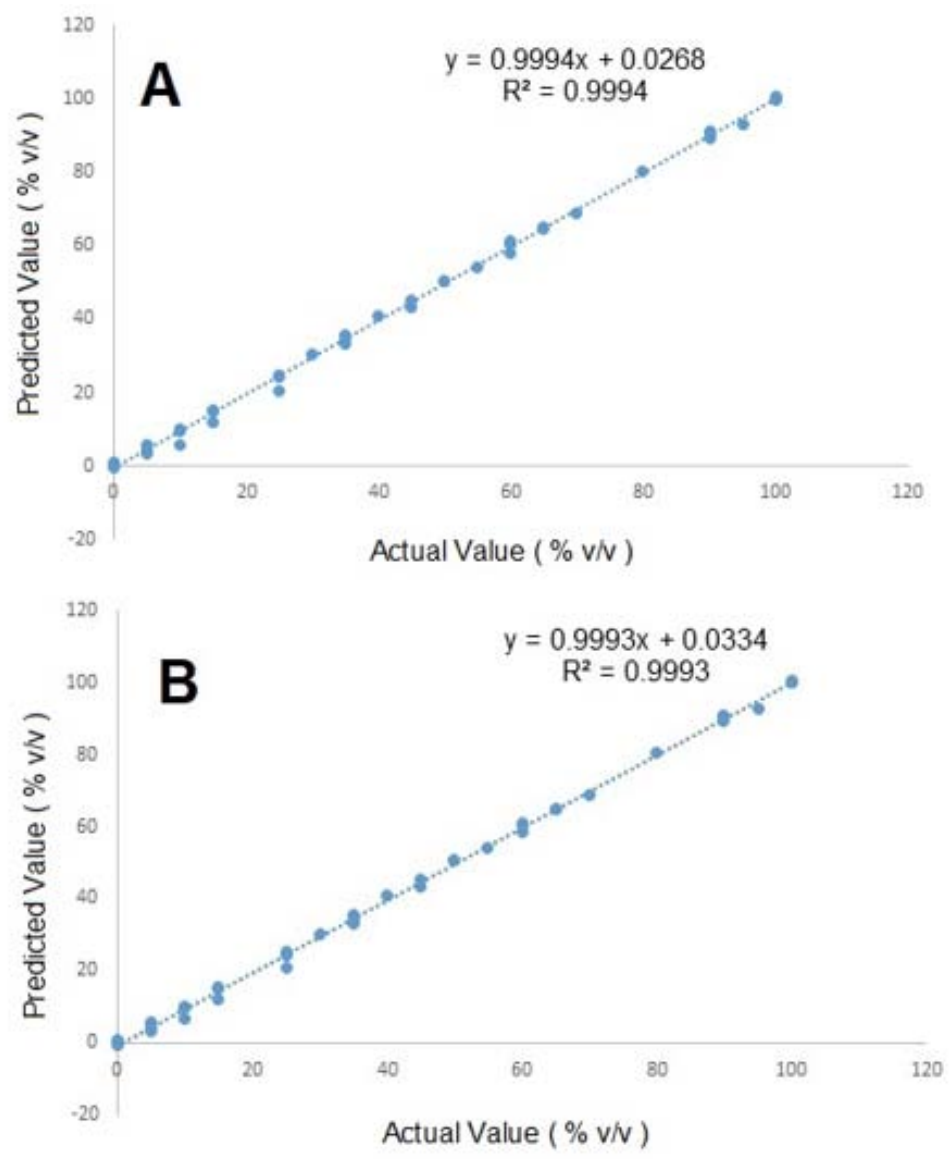

Fig. 3: Calibration model for the relationship between actual ( $x$-axis) and FTIR predicted values of patin fish oil adulterated with palm oil using partial least square $(A)$ and principle component regression $(B)$ with first derivative spectra. 


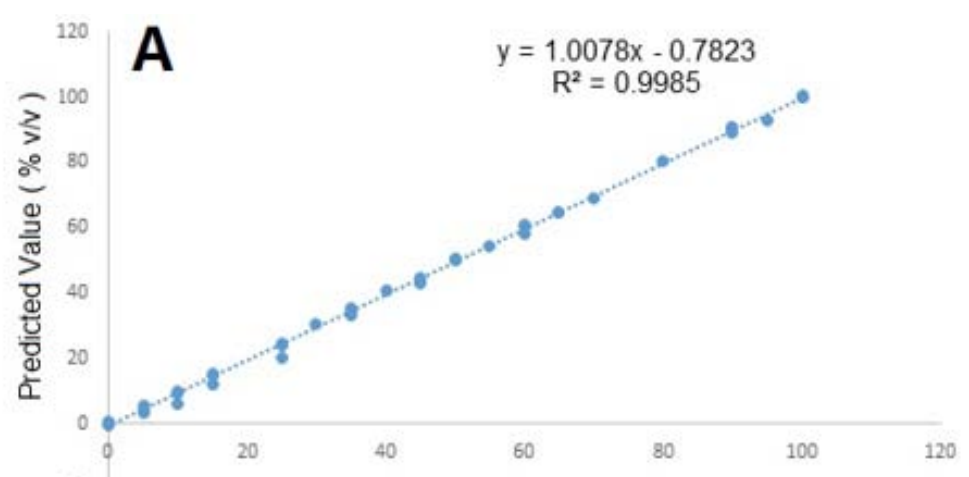

Actual Value ( $\% \mathrm{v} / \mathrm{v}$ )

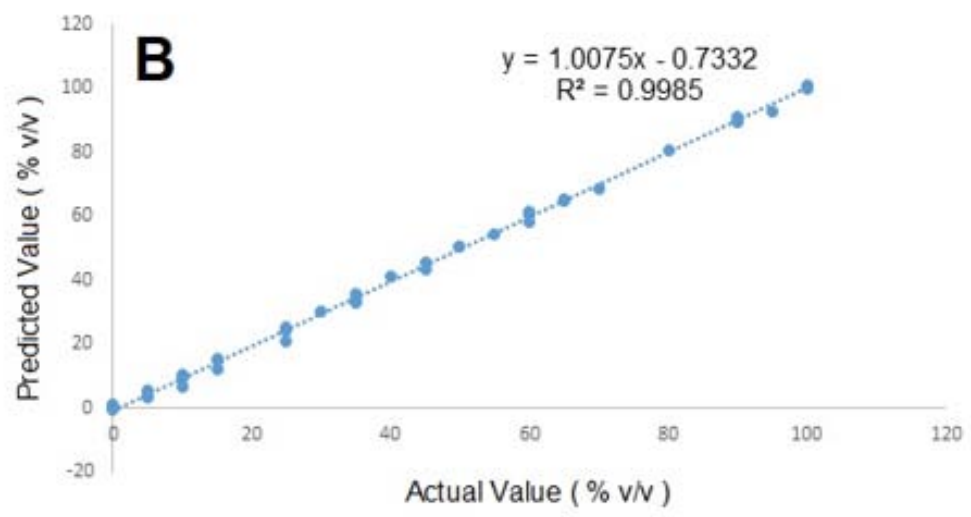

Fig. 3: The validation model for the relationship between actual ( $x$-axis) and FTIR predicted values of of patin fish oil adulterated with palm oil using partial least square (A) and principle component regression (B) with first derivative spectra

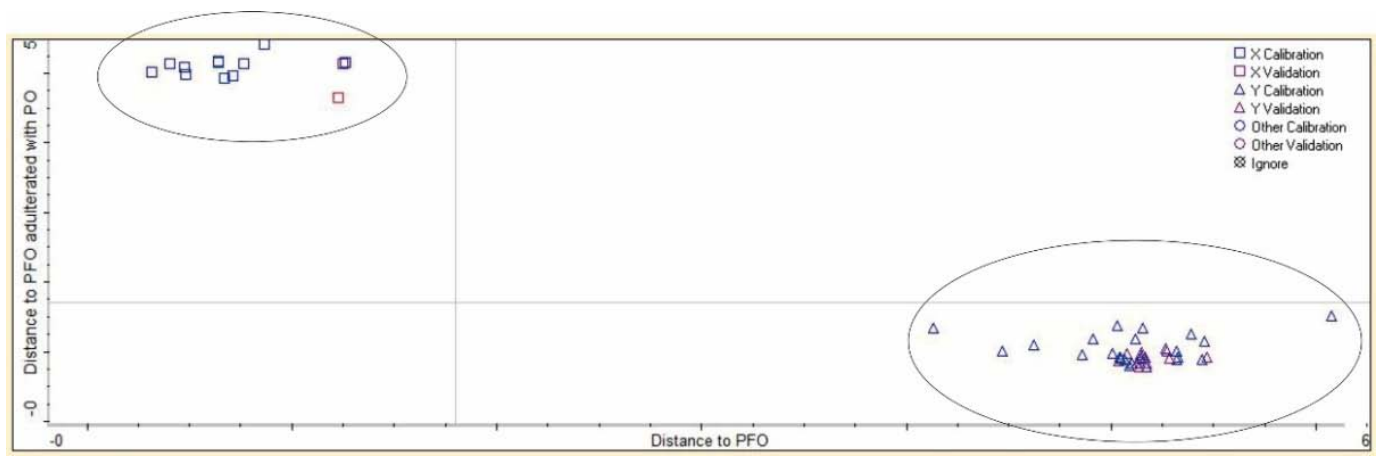

Fig. 5: The coomzans plot of patin fish oil (PFO) and palm oil (PO): $(\Delta)$ PFO adulterated with PO and ( $\square$ ) pure PFO

\section{Discriminant analysis}

Discriminant analysis is a method commonly used to decide the group between pure patin fish oil (PFO) and PFO adulterated with palm oil (PO) by computing the distance from each group center based on Mahalanobis distance units. If the grouping model have been obtained, the component of unknown samples can be predicted [15]. Discriminant analysis using the combined wavenumber regions of 1001-1501, 17761712 , and $2819-2981 \mathrm{~cm}^{-1}$ were used for classification.

The Coomans plot is the method to show the group of pure PFO and PFO adulterated with PO. This plot was obtained from calculating two principal components (PC) and then plotting the residual distances of samples from each two models [19]. Fig. 5 exhibited the Coomans Plot for pure PFO and PFO adulterated with PO. The x-axis described the Mahalanobis distance for pure PFO and the y-axis exhibited the distance of PFO adulterated with PO. The Coomans plot in fig. 5 exhibited the group division between pure PFO and PFO adulterated with PO. The DA model is capable of grouping the oils with an accuracy level of $100 \%$. Therefore, this method is a suitable method for grouping the samples and no samples were mistakenly grouped.

\section{CONCLUSION}

FTIR spectroscopy combined with chemometrics is suitable method for authentication of patin fish oil (PFO) and PFO adulterated with PO. Chemometrics method using PLS and PCR can give an accepted model for the calibration and validation with high $\mathrm{R}^{2}$ value and low RMSEC and RMSEP values using first derivative FTIR spectra in the regions at 1001-1501, 1776-1712, and 2819. $2981 \mathrm{~cm}^{-1}$. Discriminant analysis could also successfully used for grouping the pure PFO and PFO adulterated with PO with accuracy level of $100 \%$.

\section{ACKNOWLEDGMENT}

Author thanks to The Ministry of Research, Technology, and Higher Education of the Republic of Indonesia for its scholarship so that this research can be done. 


\section{AUTHORS CONTRIBUTIONS}

ARP compiled data dan prepared manuscript. AR and SR designed research, analysed data, and made critical thinking on manuscript

\section{CONFLICT OF INTERESTS}

The author declares that there is no conflict of interest

\section{REFERENCES}

1. Guil Guerrero JL, Venegas Venegas E, Rincón Cervera MA, Suarez MD. Fatty acid profiles of livers from selected marine fish species. J Food Compos Anal 2011;24:217-22.

2. Connor WE. Importance of-B fatty acids in health and disease. J Clin Nutr 2000;17:1S-5S.

3. Jicha GA, Markesbery WR. Omega-3 fatty acids: potential role in the management of early Alzheimer's disease. ClinInterv Aging 2010;5:45-52.

4. Christy AA, Kasemsumran SDuY, Ozaki Y. The detection and quantification of adulteration in olive oil by near-infrared spectroscopy and chemometrics. Anal Sci 2004;20:935-40.

5. Rohman A, Che Man YBC. Fourier transform infrared (FTIR) spectroscopy for analysis of extra virgin olive oil adulterated with palm oil. Food Res Int 2010;43:886-92.

6. Héberger K. Chemoinformatics-multivariate mathematicalstatistical methods for data evaluation. In: Medical Applications of Mass Spectrometry; 2008. p. 141-69.

7. Rohman A, Che Man YB. The use of fourier transform midinfrared (FT-MIR) spectroscopy for detection and quantification of adulteration in virgin coconut oil. Food Chem 2011;129:583-8.

8. Fadzlillah NA, Che Man YB, Rohman A. FTIR spectroscopy combined with chemometric for analysis of sesame oil adulterated with corn oil. Int J Food Prop 2014;17:1275-82.

9. Gurdeniz G, Ozen B. Detection of adulteration of extra-virgin olive oil by chemometric analysis of mid-infrared spectral data. Food Chem 2009;116:519-25.
10. Rohman A, Man YBC. The chemometrics approach applied to FTIR spectral data for the analysis of rice bran oil in extra virgin olive oil. Chemom Intell Lab Syst 2012;100:129-34.

11. Yuliani F, Riyanto S, Rohman A. Application of FTIR spectra combined with chemometrics for analysis of candlenut oil adulteration. Int J Appl Pharm 2018;10:54-9.

12. Prabowo P, Muflihah, Rohman A. Monitoring oxidative levels of frying oils using FTIR spectroscopy and multivariate calibration. Int J Appl Pharm 2018;10:82-7.

13. Maggio RM, Kaufman TS, Carlo MD, Cerretani L, Bendini A, Cichelli A, et al. Monitoring of fatty acid composition in virgin olive oil by Fourier transformed infrared spectroscopy coupled with partial least squares. Food Chem 2009;114:1549-54.

14. Paradkar MM, Irudayaraj JA. Rapid FTIR spectroscopic method for estimation of caffeine in soft drinks and total methylxanthines in tea and coffee. J Food Sci 2002;67:2507-11.

15. Ballabio D, Todeschini R. Multivariate classification for qualitative analysis. In: DW Sun (Ed). Infrared Spectroscopy for Food Quality Analysis and Control. Elsevier, London; 2009.

16. Yap KYL, Chan SY, Lim CS. Infrared-based protocol for the identification and categorization of ginseng and its products. Food Res Int 2007:40:643-52.

17. Che Man Y, Syahariza ZA, Mirghani ME, Jinap S, Bakar J. Analysis of potential lard adulteration in chocolate and chocolate products using fourier transform infrared spectroscopy. Food Chem 2005;90:815-9.

18. Smith B. Quantitative spectroscopy: theory and practice. $1^{\text {st }}$ ed. Academic Press: America; 2002.

19. Gurdeniz G, Ozen B. Detection of adulteration of extra-virgin olive oil by chemometric analysis of mid-infrared spectral data Food Chem 2009;116:19-525.

20. Guillén MD, Cabo N. Characterization of edible oils and lard by fourier transform infrared spectroscopy. Relationships between composition and frequency of concrete bands in the fingerprint region. J Am Oil Chem Soc 1997;74:1281-6. 